\title{
One-Pot Synthesis and Characterization of Biomimetic Copper(I) Complexes
}

Isabelle Gerz, ${ }^{a, b}$ Chiara Negri, ${ }^{a, b}$ David Wragg, ${ }^{a, b}$ Sigurd Øien-Ødegaard, ${ }^{a, b}$ Mats Tilset, ${ }^{a, b}$ Unni Olsbye, ${ }^{a, b}$ Mohamed Amedjkouh ${ }^{a, b} *$

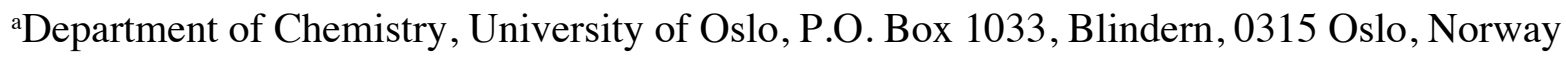

${ }^{\mathrm{b} C e n t e r}$ for Materials Science and Nanotechnology (SMN), Faculty of Mathematics and Natural Sciences, University of Oslo, P.O. Box 1126, Blindern, 0318 Oslo, Norway

E-Mail: mamou@kjemi.uio.no

ABSTRACT: A modular one-pot synthesis yielding tetracoordinated- $\mathrm{N}, \mathrm{N}, \mathrm{N}, \mathrm{N}-\operatorname{copper}(\mathrm{I})$ complexes bearing imine and diimine ligands was developed. Copper aids the condensation of a pyridine or imidazole carbaldehyde with a biphenyl amine and even stabilizes labile ligands. Tetradentate and bidentate ligands were formed, the latter forming homoleptic $\mathrm{CuL}_{2}$ complexes. The identity and purity of the compounds were assessed by NMR, elemental analysis and mass spectrometry. The interconversion of different species in solution was studied by variable temperature NMR. The complexes aim to mimic the histidine copper brace of lytic polysaccharide monooxygenases (LPMOs) and bond lengths obtained from XAS and single crystal XRD for the complexes were compared to reported photoreduced LPMOs. 
INTRODUCTION: When it comes to tuning the selectivity of oxidations, enzymes can serve as blueprints for catalysts. ${ }^{1}$ Monooxygenases are, as specified by their name, a class of enzymes that can add a single hydroxyl group to their substrate. Two prominent examples of this class utilize copper as a cofactor: the particulate methane monooxygenase (pMMO) and the lytic polysaccharide monooxygenase (LPMO). They both feature a structural motif known as histidine brace, coordinating a copper atom. ${ }^{2-6}$ While this structural motif is the sole active site in LPMOs, its role in pMMO is debated, ${ }^{6}$ motivating the search for small molecule models for the histidine copper brace. If the model behaves analogously in spectroscopy and catalysis, a better understanding of the active sites of LPMOs and pMMO is attainable.

Approaches from this biomimetic angle have resulted in a variety of $\mathrm{N}$-coordinated copper complexes. Prominent examples combine aliphatic amines, amides or imines with heterocycles in the ligand (examples in Figure 1).$^{7-9}$ The most commonly employed heterocycles are pyridines and imidazoles. ${ }^{10}$ We herein report copper complexes with a tetra-N-coordinated copper center. The heterocycle (either imidazole or pyridine) is connected to the biphenyl backbone through an imine - creating a 1,4-relationship between the coordinating nitrogen atoms. Similar complexes have either been made by ligand synthesis and subsequent metalation, ${ }^{11-13}$ or a one-pot synthesis. ${ }^{14-}$ ${ }^{16}$ The biphenyl moiety of the complexes introduces a versatile backbone to tune the steric and electronic attributes of the system by established methods of organic chemistry. Notably, it introduces a possible anchor point for immobilization without strongly restraining the coordination geometry, as opposed to e.g. diamines on the same aromatic ring. 


\section{LPMO Histidine Brace:}

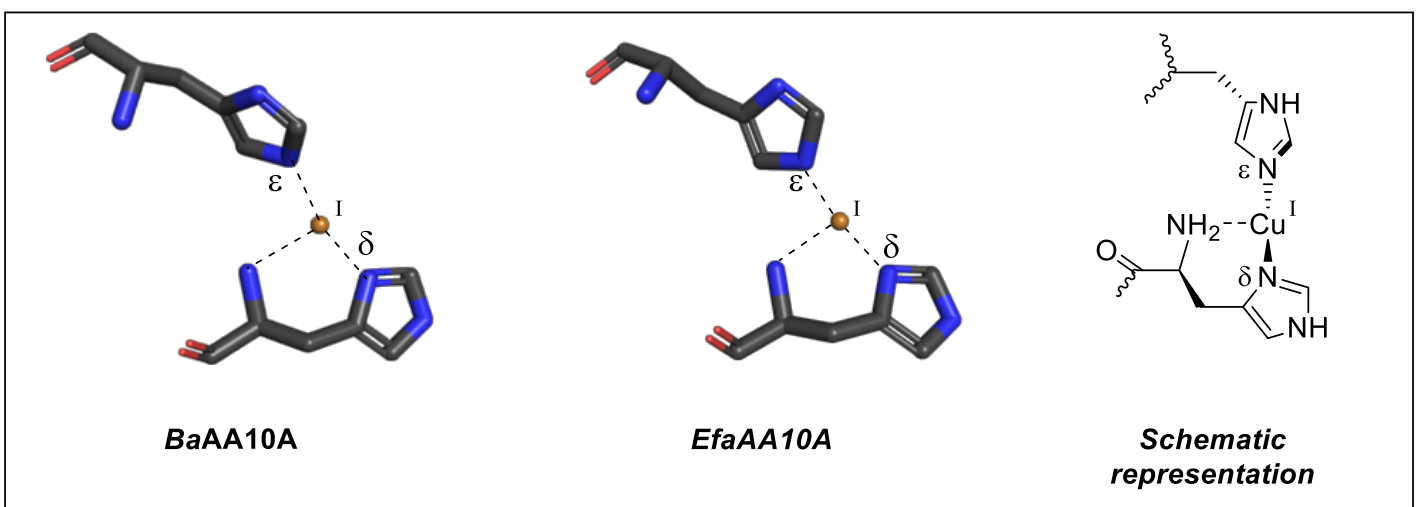

Mimetic Complexes:

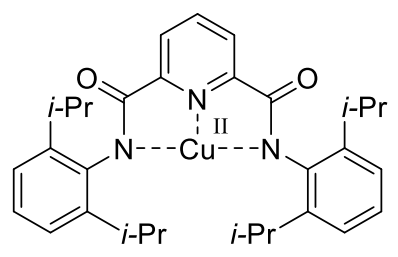

Tolman 2010

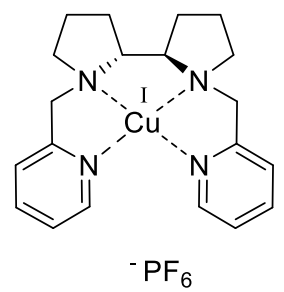

Garcia-Bosch 2016

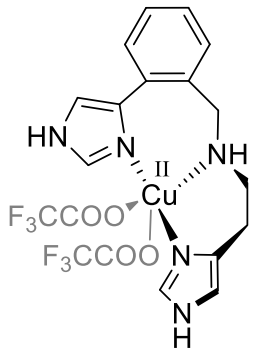

Itoh 2020

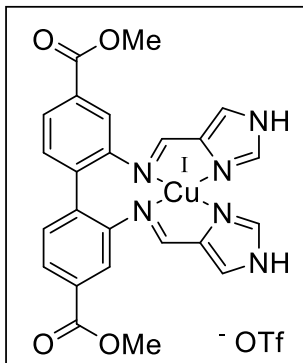

This work

Figure 1. The histidine brace motif: The top row shows the histidine copper brace in two photoreduced fungal LPMOs: BaAA10A (PDB-code: 2YOX; AA10 enzymes were previously referred to as CBM33), reported by Hemsworth et al., EfaAA10A (PDB-code: 4ALT), reported by Gudmundsson et al., and their schematic representation. ${ }^{3,17}$ Below, a selection of the many mimics of the active site in different oxidation states..$^{9,18,19}$

Biomimetic complexes reported in literature have been reported as either copper(I) or copper(II) compounds, the latter being more common. The characterization of copper(II) complexes relies heavily on crystallization, as single crystal XRD often provides the strongest support for their structure. Studying the complex in its copper(I) state allows to observe it by NMR in solution, 
given that oxidation is prevented. By working in rigorously oxygen free surrounding, we were able to characterize the complexes and study their stability over time.

RESULTS: The apparent approach to obtain target $\mathbf{1}$ was to synthesize the ligand and subsequently metalate it with a copper(I) salt. Initially, it was attempted to synthesize the tetradentate ligand for $\mathbf{1}$ by acid-catalyzed condensation of $1 H$-imidazole-4-carbaldehyde with dimethyl 2,2'-diaminobiphenyl-4,4'-dicarboxylate under reflux, which showed incomplete conversion. Subsequent work-up attempts failed due to the lability of the diimine during chromatography, even with a basic eluent. In an effort to stabilize the diimine, condensation and metalation were attempted in one step (see Scheme 1), providing complex $\mathbf{1}$. The synthesis is an overnight reaction at ambient temperature in acetonitrile. The minimalistic work-up consists of filtration of the solid product and a washing step.
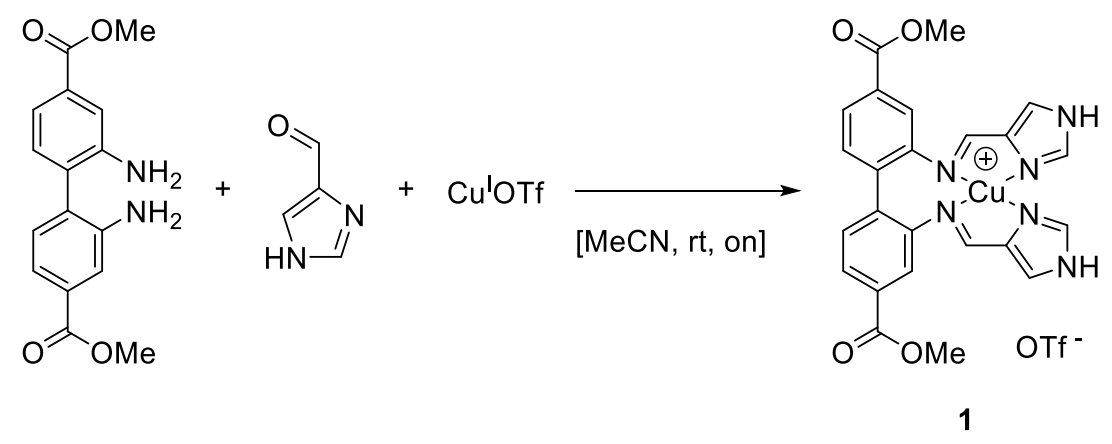

Scheme 1. Synthesis of 1.

Copper complex 1 was isolated in good yields and high purity. The complex was characterized by NMR, HRMS and elemental analysis. The ${ }^{1} \mathrm{H}$ NMR spectrum of $\mathbf{1}$ shows six resonances in the aromatic region, each integrating for two protons. Additionally, there is one resonance at $13.35 \mathrm{ppm}$ (imidazole $\mathrm{NH}$, two protons) and one at $3.83 \mathrm{ppm}$ (methyl group, six protons). The spectrum is bare of the aldehyde and $\mathrm{NH}_{2}$ resonances found in the starting materials - instead, a resonance consistent with an imine was observed $(8.31 \mathrm{ppm})$, correlating to a carbon resonance at 4 
$154.5 \mathrm{ppm}$ in an HSQC experiment. The copper(II) complex corresponding to $\mathbf{1}$ was synthesized from $\mathrm{Cu}(\mathrm{OTf})_{2}$. The complex was characterized by elemental analysis, UV/Vis and HRMS (see SI, compound 1b). It showed an absorbance band at $670 \mathrm{~nm}\left(103.97 \mathrm{~L} \cdot \mathrm{mol}^{-1} \mathrm{~cm}^{-1}\right)$, comparable to literature reports of similar $\mathrm{Cu}(\mathrm{II})$ complexes. ${ }^{16}$ Due to its paramagnetic nature, complex $\mathbf{1 b}$ has extremely broadened peaks that merge into the baseline in ${ }^{1} \mathrm{H}$ NMR. Ascorbic acid is commonly employed as a reductant for LPMOs. ${ }^{20}$ Complex $\mathbf{1 b}$ was reduced to complex $\mathbf{1}$ with ascorbic acid in an NMR experiment, showing that the synthesis yields the same ligand regardless of the oxidation state of the copper salt (Figure S7).

The synthesis method depicted in Scheme 1 was extended to five more copper(I) complexes (26) (Figure 2).

A: ML<smiles>COC(=O)c1ccc(-c2ccc(C(=O)OC)cc2N(Cc2c[nH]cn2)Cc2c[nH]cn2)c(N(Cl)Cc2cnc[nH]2)c1</smiles>

B: $M L_{2}$

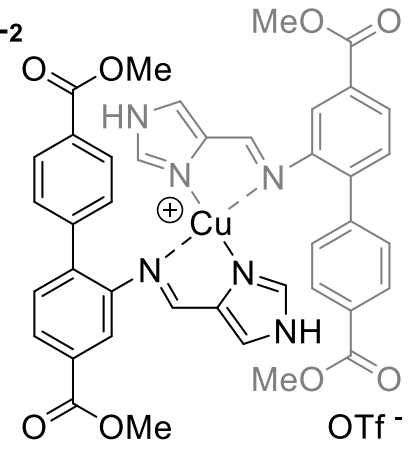

2<smiles>COC(=O)c1ccc(-c2ccc(C(=O)OC)cc2N(C=Nc2ccccn2)Cc2ccccn2)c(N=Cc2ccccn2)c1</smiles>

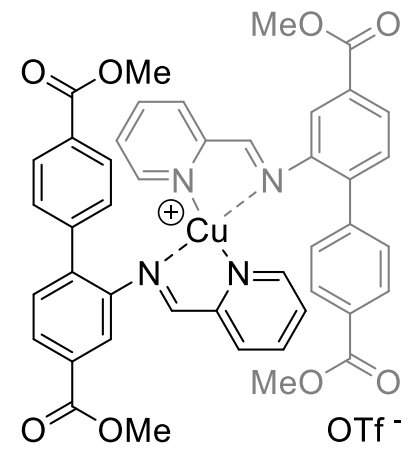

4

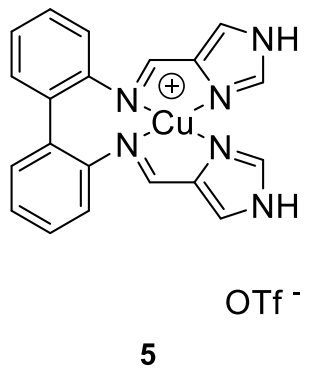

5

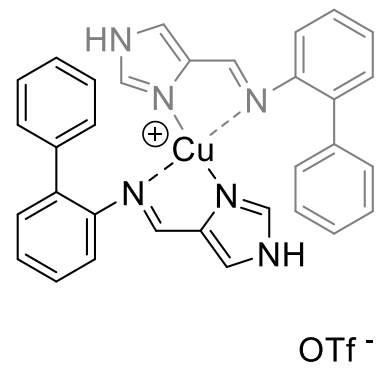

6

Figure 2. Synthesized copper(I) complexes. A: Complexes of the type ML bear tetradentate ligands. B: Homoleptic $\mathrm{ML}_{2}$ complexes with bidentate ligands. 
For all of them, MS showed an $\mathrm{m} / \mathrm{z}$ consistent with the complex cation charged +1 . The complexes were isolated in high purities (demonstrated by elemental analysis) and in fair to good yields. Except for compound 3, all compounds could be studied well by NMR. The NMR studies were conducted in flame-sealed tubes. As described earlier for $\mathbf{1}$, the imine resonance is easily identified with the HSQC experiment. With further 2D NMR analysis, it was possible to assign proton and carbon signals for complexes 1, 2 and 4-6. The resulting tetracoordinated complexes can be divided into two groups. The first group $(\mathbf{1}, \mathbf{3}$ and $\mathbf{5}$, Figure $2 \mathrm{~A})$ is of the type ML, where $\mathrm{L}$ is a tetradentate ligand. The other group (Figure 2B) consists of complexes 2, 4 and $\mathbf{6}$. Starting from 2-aminobiphenyls, the formation of $\mathrm{ML}_{2}$ ( $\mathrm{L}$ is a bidentate ligand) complexes was consistently observed by NMR, MS and elemental analysis. For compound $\mathbf{2}$ and $\mathbf{4}$, single crystal structures showed the coordination of two bidentate ligands (Figure 6, Figure S34). The counterion does not coordinate, but for $\mathbf{2}$ a hydrogen bond to the imidazole-NH was noted. It was found that homoleptic complexes were favored regardless of the equivalents used (one or two equivalents of aldehyde and 2-aminobiphenyl relative to the copper salt). The preferential formation of $\mathrm{ML}_{2}$ complexes has been reported before for bi- and tridentate ligands that coordinate through nitrogen to copper(I) ${ }^{21,22}$ For the NMR studies of both groups of complexes, the solvent choice affected not only the broadness and shift of the resonances in NMR spectroscopy but also the complexes' stability in solution. DMSO dissolves all complexes, while the solubility in acetonitrile is limited. The $\mathrm{ML}_{2}$ complexes are generally more soluble than the ML complexes. 


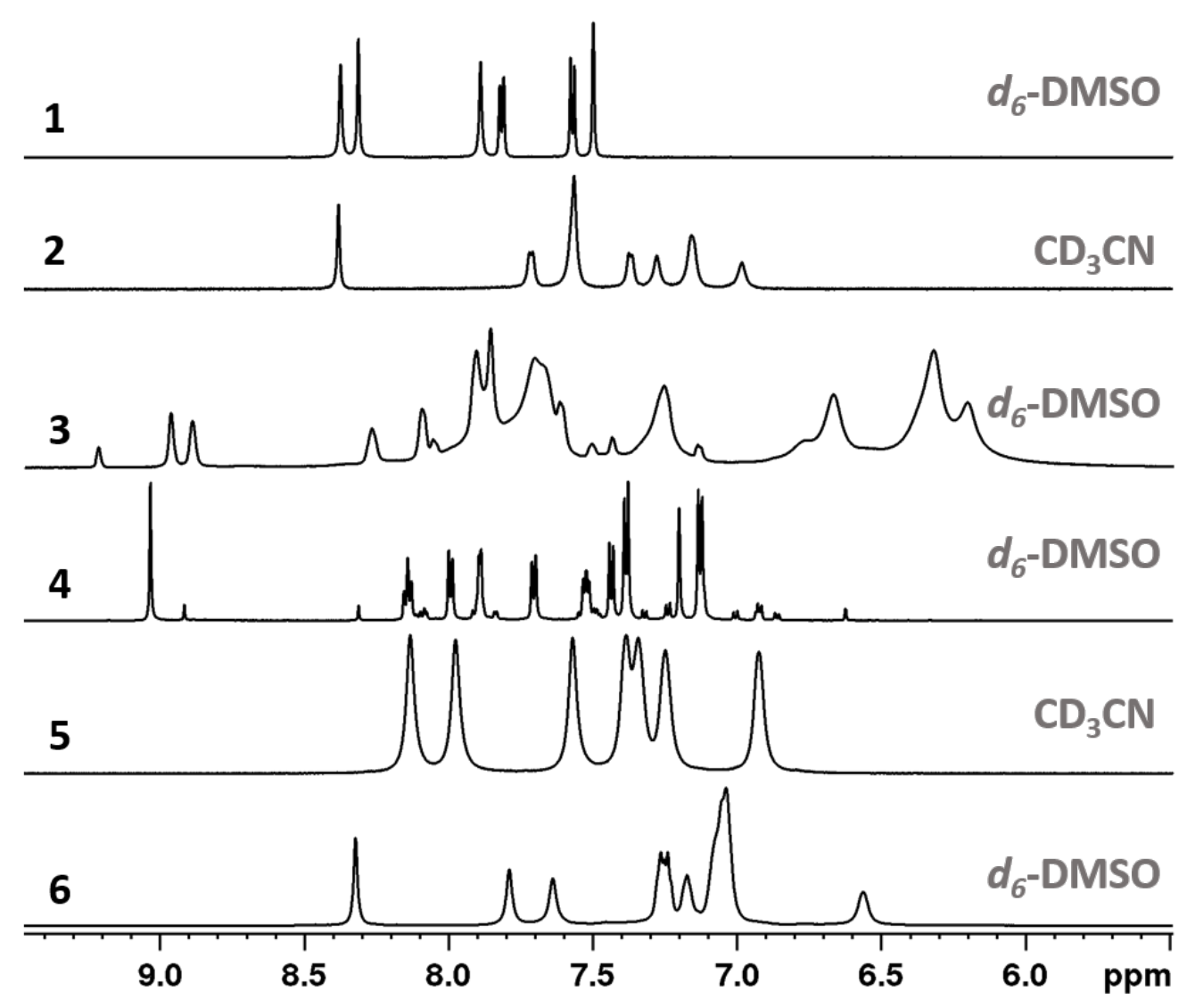

Figure 3. Aromatic region of the ${ }^{1} \mathrm{H}$ NMR spectra $(600 \mathrm{MHz}, 295 \mathrm{~K})$ of the copper(I) complexes synthesized in this work.

The NMR spectrum of $\mathbf{1}$ shows a narrow peak-width, comparable to organic molecules (Figure $\mathbf{3}$, top spectrum). In $d_{6}$-DMSO, 4 had an equally narrow peak-width, but a secondary, minor species was observed. Changing the solvent to acetonitrile resulted in pronounced changes in the NMR spectrum, as discussed for the variable temperature experiment for $\mathbf{4}$ below. Compound 5's 'H NMR resonances were broader in $d_{6}$-DMSO (Figure S26) than in acetonitrile. Compound 2 is less stable in DMSO than in acetonitrile. The peak broadness of compounds $\mathbf{2}, \mathbf{3}, \mathbf{5}$ and $\mathbf{6}$ in ${ }^{1} \mathrm{H}$ NMR masks smaller couplings. Possible explanations for the broadening in the spectra are rapid changes in ligation and/or conformation. The ligation change might occur for example through coordination of the solvent or intermolecular ligand exchange. Both NMR solvents employed in this study, 
acetonitrile and DMSO, are coordinating solvents. We observed that complexes with considerable line broadening decomposed during NMR analysis. A sealed NMR sample of 1 remained unchanged even after months, while $\mathbf{2 , 4 , 5}$, and $\mathbf{6}$ partially decomposed into the starting materials during longer NMR experiments. A juxtaposition of the ${ }^{1} \mathrm{H}$ NMR spectra of the complexes after prolonged NMR experiments and the amines they were synthesized from shows good agreement between the decomposition peaks and the amine for all four complexes (Figure S33). In combination with the simultaneous rise of an aldehyde peak, it is evident that the decomposition can be attributed to hydrolysis of the imine bond. The imine is more prone to hydrolysis in the free ligand than in the coordinated ligand, as seen during synthesis. It seems likely that the $\mathrm{ML}_{2}$-type complexes 2, 4 and $\mathbf{6}$ are in equilibrium with an ML complex and the free ligand (exemplified for 2 in Figure 4). This is supported by the observation of all three species $\left(\mathrm{ML}_{2}, \mathrm{ML}\right.$ and free ligand) in the mass spectra of these complexes (see SI).

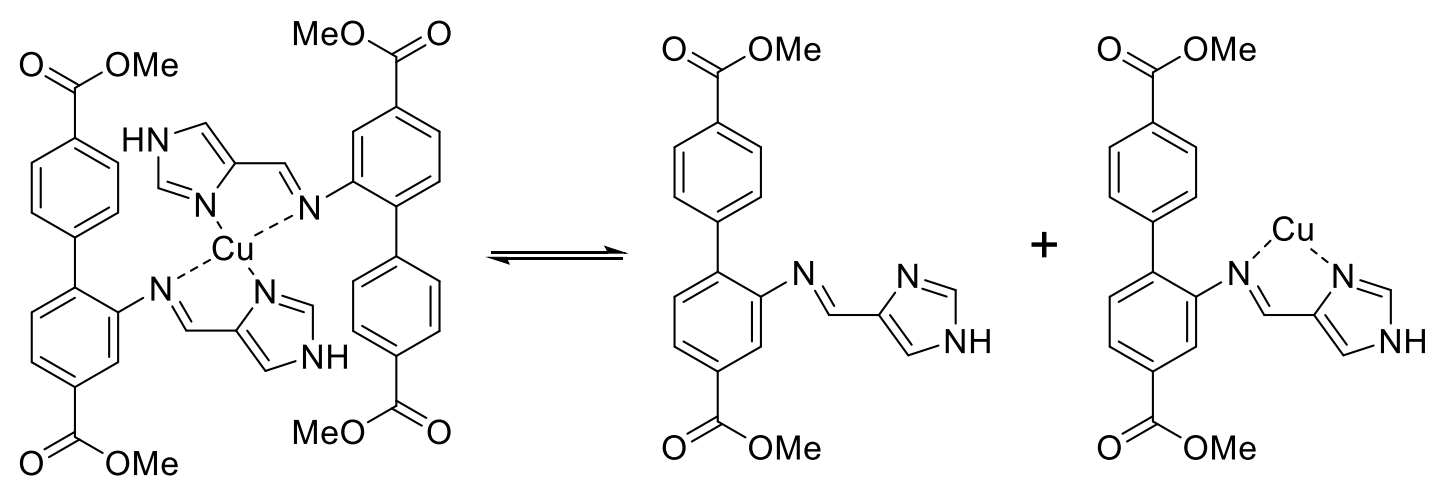

Figure 4. Equilibrium of $\mathrm{ML}_{2}$ complex 2 with the free ligand and the resulting ML complex. The ML complex might have solvent molecules as additional ligands.

The comparable broadening and decomposition that were observed for $\mathbf{5}$ are more difficult to rationalize, as a tetradentate ligand is expected to have a stronger coordination. The methyl ester 
groups are the only structural difference between $\mathbf{1}$ and $\mathbf{5}$ - despite being rather remote from the coordination center, they seem to strongly affect the solution behavior and stability of the copper complex.

The ${ }^{1} \mathrm{H}$ NMR spectra of $\mathbf{3}$ and $\mathbf{4}$ in $\mathrm{d}_{6}$-DMSO show multiple species (Figure 3), while elemental analysis and MS measurements were consistent with the structures in Figure 2. This discrepancy prompted us to investigate the ${ }^{1} \mathrm{H}$ NMR of 4 at various temperatures (Figure 5). To lower the temperature without freezing the sample, the experiment was conducted in acetonitrile.

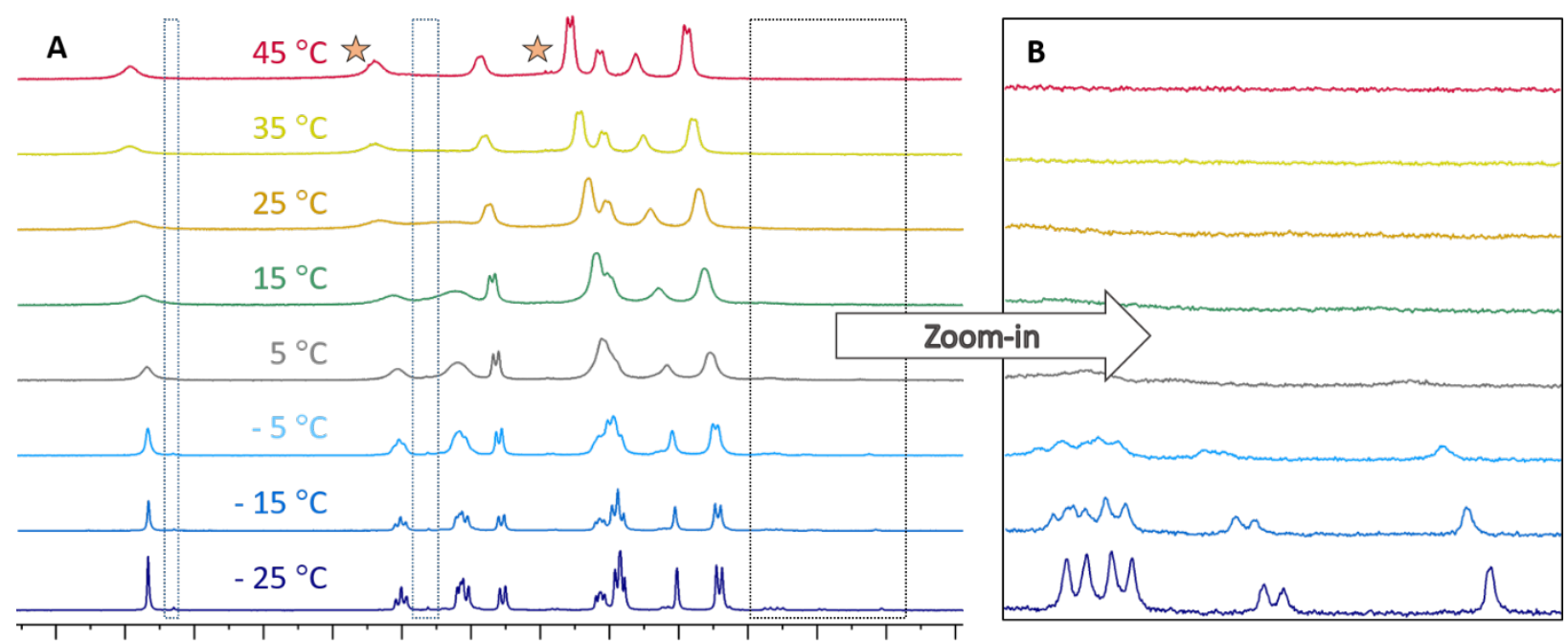

Figure 5. A: Aromatic region of the ${ }^{1} \mathrm{H}$ NMR spectra of 4 at different temperatures $\left(\mathrm{CD}_{3} \mathrm{CN}\right.$, $500 \mathrm{MHz}$ ). Stars indicate decomposition at higher temperatures. Grey boxes indicate some of the areas where the minor species is observable at low temperatures. B: The rightmost box is displayed with higher intensities for all spectra to improve visibility of the additional peaks.

The change in solvent altered the appearance of $\mathbf{4}$ at room temperature: Instead of multiple species, a single species with broadened peaks was observed (Figure $5 \mathrm{~A}, 25^{\circ} \mathrm{C}$ ). The sample was cooled down to $-25^{\circ} \mathrm{C}$ in increments of $10{ }^{\circ} \mathrm{C}$. This resulted in narrower peaks and the evolution of additional peaks (boxes in Figure 5), similar to the room temperature measurement in $\mathrm{d}_{6}$-DMSO. 
A NOESY experiment at the lowest temperature $\left(-25^{\circ} \mathrm{C}\right)$ revealed chemical exchanges between these new peaks and the major ones (see S21 and S22). These processes, which we were not able to identify unambiguously, were reversible: the temperature was increased step-wise to ambient temperature, and the spectra acquired were identical to those during the cool-down process. Elevating the temperature above room temperature also led to peak narrowing. However, minor signs of decomposition were observed (stars in Figure 5). The decomposition peaks persisted when the temperature was lowered back to room temperature. Otherwise, the spectrum regained its initial appearance. The line broadening of complexes $2,4,5, \mathbf{6}$ being accompanied by decomposition hints towards intermolecular ligand exchange as one possible underlying mechanism. Ligand exchange is well studied for heteroleptic copper complexes, as it causes the disproportionation of heteroleptic photosensitizers into less active homoleptic complexes. ${ }^{23,24}$ For homoleptic complexes, the process creates no net-change, as the product is equivalent to the complex before exchange. However, if the ligand exchange takes place via the free ligand it may open a possible pathway for decomposition (as discussed above). Decomposition was not observed in solids stored under an inert atmosphere for over a year.

It was possible to obtain crystals suitable for single crystal XRD analysis by recrystallizing $\mathbf{4}$ from $\mathrm{MeCN} /$ toluene (Figure 6). The tetracoordinated environment surrounding copper is an intermediate geometry between square-planar and tetrahedral, as determined by the structure's $\tau_{4}{ }^{\prime}$-value $\left(\tau_{4}{ }^{\prime}=0.50\right) . \tau_{4}{ }^{\prime}$ is a geometry index for tetracoordinated complexes that adapts values between 0 (square planar) and 1 (tetrahedral) ${ }^{25.26}$ The imine-N-copper bonds (N1a-Cu: 2.085(2) $\AA$;

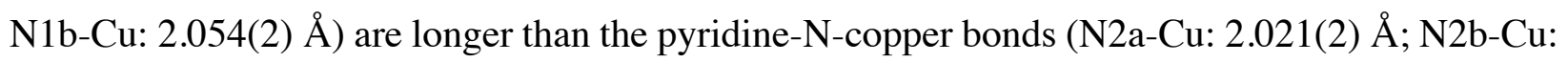
2.028(2) ̊̊). 


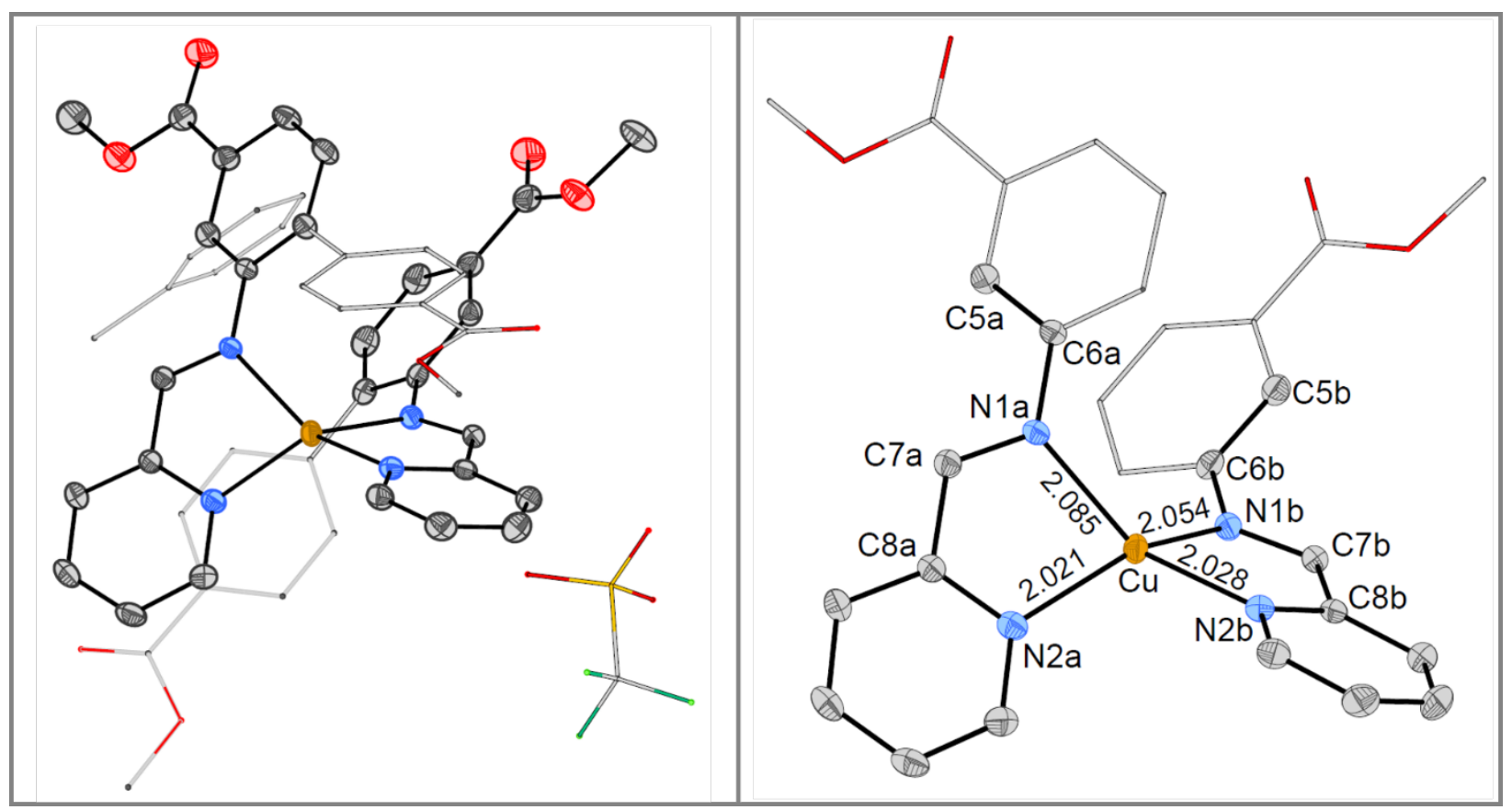

Figure 6: ORTEP-plot of 4 (ellipsoids at $50 \%$ probability). The asymmetric unit contains the complex cation, the non-coordinating anion and a solvent molecule (toluene). Hydrogen atoms are omitted and parts of the structure are represented as sticks for clarity. Selected bond lengths:

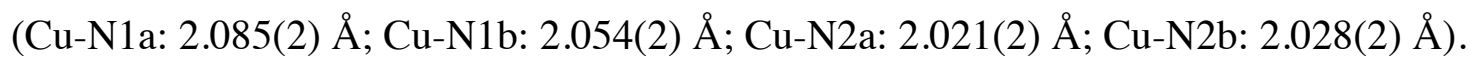

The crystal structure of $\mathbf{4}$ shows surprisingly large differences between the two ligands (despite their identical connectivity). For one, the bond lengths to copper differ not only between the type of nitrogen but also between the two ligands, creating an asymmetric environment around copper (see Figure 6, right side). The asymmetry is even clearer when comparing the dihedral angles between the imine and the adjacent phenyl ring, which were measured to be $27.109(9){ }^{\circ}(\mathrm{C} 5 \mathrm{a}-\mathrm{C} 6 \mathrm{a}-$ $\mathrm{N} 1 \mathrm{a}-\mathrm{C} 7 \mathrm{a})$ and $42.755(9)^{\circ}(\mathrm{C} 5 \mathrm{~b}-\mathrm{C} 6 \mathrm{~b}-\mathrm{N} 1 \mathrm{~b}-\mathrm{C} 7 \mathrm{~b})$ respectively. This type of discrimination between the two ligands should integrate 1:1 in ${ }^{1} \mathrm{H}$ NMR or show an averaged peak. However, the variable temperature NMR experiment discussed above does not support this hypothesis, as a major and one or more minor species were observed. It is therefore likely that the observed conformation in 
single crystal XRD is a property of its solid state and does not necessarily represent the conformation in solution.

XAS spectra of complexes $\mathbf{1}$ and $\mathbf{2}$ were collected (measured neat in a capillary). They were very similar to each other (see SI), pointing to an alike coordination surrounding copper. Both spectra indicated the presence of a light atom at around $1.9 \AA$ (1.94(2) $\AA$ for $\mathbf{1}, 1.92(6) \AA$ for 2$)$. The data were not consistent with four nitrogen atoms in the first shell. We presume that copper binds tighter to imidazole at the expense of the copper-imine bonds - resulting in a lower coordination number in the first shell. This hypothesis is supported by a preliminary single crystal XRD structure of compound 2. The bond lengths between the coordinating nitrogens and copper are listed in

\section{Table 1.}

Hemsworth et al. and Gudmundsson et al. have presented structural data for non-fungal LPMOs (AA10) in their photoreduced copper(I) state (see Figure 1).,17 As the oxidation state influences coordination number, coordination geometry and bond lengths of the active center, we focus our comparative analysis on these structures. Despite variation between the two structures (the bond lengths in $\mathrm{Ba}$-CBM33 seem to be longer in general), it appears that the amino-copper bond is about $0.3 \AA$ longer than the imidazole-nitrogen-copper bond. A major difference between the enzyme's active site and the herein presented mimics is the absence of a coordinating amine. Instead, two imines coordinate to the copper center.

Table 1. Bond lengths in single crystal XRD studies of photoreduced AA10 LPMO active sites and two of the $\mathrm{Cu}(\mathrm{I})$ complexes presented in this work.

\begin{tabular}{l|l|l}
\hline & $\begin{array}{l}\text { Bond Length of Copper to N- } \\
\text { Heterocycle }\end{array}$ & $\begin{array}{l}\text { Bond Length of Copper to } \\
\text { Amine/ Imine }\end{array}$ \\
\hline
\end{tabular}




\begin{tabular}{l|l|l}
\hline EfaCBM33A ${ }^{17}$ & $1.91 \AA(\mathrm{N} \varepsilon), 1.94 \AA(\mathrm{N} \delta)$ & $2.19 \AA$ \\
\hline$B a-\mathrm{CBM} 33$ & $2.01 \AA(\mathrm{N} \varepsilon), 1.98 \AA(\mathrm{N} \delta)$ & $2.31 \AA$ \\
\hline $\mathbf{2}$ & $1.93 \AA ; 1.92 \AA$ & $2.32 \AA ; 2.21 \AA$ \\
\hline $\mathbf{4}$ & $2.03 \AA, 2.02 \AA$ & $2.09 \AA, 2.05 \AA$ \\
\hline
\end{tabular}

* The asymmetric unit contains two complexes.

The pyridine based structure $\mathbf{4}$ does not represent the bond lengths in the enzyme very well, as all four bond lengths are between 2.0 and 2.1 Å. Structures based on imidazole should be better mimics, as histidine itself is an imidazole derivative. The XAS data indicates that there is indeed a coordinating atom to be found at a distance comparable to the imidazole-copper bond-length in the copper(I) LPMO structures. Further analysis is needed to conclude if an imine can be a surrogate for an amine group in this context.

CONCLUSION AND FUTURE OUTLOOK: The modular approach of the protocol allowed synthesizing the desired N-coordinated copper complexes. The one-pot synthesis avoids timeconsuming work-ups and stabilizes the imine ligand. All complexes we present have a biphenyl backbone, on which some bear two linearly arranged ester groups. After the esters' hydrolysis, these complexes can function as linkers in a MOF, e.g. UiO-67. This possible immobilization paves the way for applications beyond modelling the enzyme, such as heterogeneous catalysis. Neither ligand nor complex can be expected to withstand the harsh conditions of MOF synthesis. Therefore, compatibility with protocols for post-synthetic linker modification is crucial for the intended immobilization in a MOF. To the best of our knowledge, the herein presented synthesis conditions can be tolerated by UiO-67. 
Future work will test if the biomimicry of these complexes extends to their reactivity. It is of interest how they react towards oxygen. We further want to investigate whether they oxidize $\mathrm{C}-\mathrm{H}$ bonds and if they do so selectively.

EXPERIMENTAL: All syntheses involving $\mathrm{Cu}(\mathrm{I})$ species were carried out in an Ar-filled UNIlab pro glovebox from MBraun. All solvents used were degassed with the freeze-pump-thaw technique (3 cycles). CuOTf, $\mathrm{CuOTf}_{2}$, the respective aldehydes, biphenyl-2,2'-diamine and biphenyl-2-amine were purchased from commercial sources and used as received. Dimethyl 2aminobiphenyl-4,4'-dicarboxylate and dimethyl 2,2'-diaminobiphenyl-4,4'-dicarboxylate were synthesized according to literature..$^{27,28}$

MS (ESI) was recorded on a Bruker maXis II ETD spectrometer by Principal Engineer Osamu Sekiguchi. UV/Vis measurements were performed on a Specord 200 Plus instrument. Elemental analysis was performed by Mikroanalytisches Laboratorium Kolbe, Oberhausen, Germany. Single crystal diffraction data were acquired on a Bruker D8 Venture equipped with a Photon 100 detector, and using Mo $\mathrm{K} \alpha$ radiation $(\lambda=0.71073 \AA$ ) from an Incoatec i $\mu \mathrm{S}$ microsource. Data reduction was performed with the Bruker Apex3 Suite, the structures were solved with ShelXT and refined with ShelXL. ${ }^{29,30}$ Olex2 was used as the user interface. ${ }^{31}$ The cif files were edited with enCIFer v. 1.4..$^{32}$ and molecular graphics were produced with Diamond v. 4.6.2. 


\section{General procedure:}

[Group A: The diamine (100 mg), CuOTf (1 equiv.) and the respective aldehyde (2 equiv). were stirred overnight in acetonitrile $(2 \mathrm{~mL})$.][Group B: The amine (100 mg), CuOTf (1/2 equiv.) and the respective aldehyde ( 1 equiv.) were stirred overnight in acetonitrile ( $2 \mathrm{~mL}$ ).] If necessary, the product was precipitated by addition of $\mathrm{Et}_{2} \mathrm{O}$. The solids were collected through filtration and washed with acetonitrile/diethyl ether (depending on solubility). The solid was dried under vacuum.

The authors thank Elijah Aller for developing an adapter to fabricate sealed NMR tubes for airsensitive compounds to be measured in solvents with high boiling points. We thank Knut T. Hylland for scientific discussions and assistance with the temperature NMR experiment. IG furthermore thanks him and Kristian Sørnes for providing starting material. CN is grateful to Dr. Eliza Borfecchia for the fruitful scientific discussions on EXAFS analysis. We acknowledge Stian Svelle, Evgeniy Redekop, Karoline Kvande, Lars Fahl Lundegaard, Robin Christensen, Konstantin Klementiev, Justus Just and Matteo Ciambezi for contributing to XAS measurements.

This project has received funding from the European Research Council (ERC) under the European Union's Horizon 2020 research and innovation program (grant agreement No 856446 (CUBE)). We acknowledge MAX IV Laboratory for time on Beamline Balder under Proposal 20190347. Research conducted at MAX IV, a Swedish national user facility, is supported by the Swedish Research council under contract 2018-07152, the Swedish Governmental Agency for Innovation Systems under contract 2018-04969, and Formas under contract 2019-02496. This work was partly supported by the Research Council of Norway through the Norwegian NMR 
Package in 1994 and partly supported by the Research Council of Norway through the Norwegian NMR Platform, NNP (226244/F50).

\section{CONFLICTS OF INTEREST}

There are no conflicts to declare.

\section{ASSOCIATED CONTENT}

The crystal structures are submitted to The Cambridge Crystallographic Data Centre with identifiers CCDC 2048291-2048292 and can be obtained free of charge from http://www.ccdc.cam.ac.uk/cgi-bin/catreq.cgi. The following files are available free of charge.

Crystal details for X (CIF)

Crystal details for Y (CIF)

\section{AUTHOR INFORMATION}

Corresponding author

*E-mail: mamou@kjemi.uio.no. Phone: +47-22857009. 


\section{REFERENCES:}

(1) Engelmann, X.; Monte-Pérez, I.; Ray, K. Oxidation Reactions with Bioinspired Mononuclear Non-Heme Metal-Oxo Complexes. Angew. Chem. Int. Ed. 2016, 55 (27), 7632-7649. https://doi.org/10.1002/anie.201600507.

(2) Quinlan, R. J.; Sweeney, M. D.; Leggio, L. L.; Otten, H.; Poulsen, J.-C. N.; Johansen, K. S.; Krogh, K. B. R. M.; Jørgensen, C. I.; Tovborg, M.; Anthonsen, A.; Tryfona, T.; Walter, C. P.; Dupree, P.; Xu, F.; Davies, G. J.; Walton, P. H. Insights into the Oxidative Degradation of Cellulose by a Copper Metalloenzyme That Exploits Biomass Components. PNAS 2011, 108 (37), 15079-15084. https://doi.org/10.1073/pnas.1105776108.

(3) Hemsworth, G. R.; Taylor, E. J.; Kim, R. Q.; Gregory, R. C.; Lewis, S. J.; Turkenburg, J. P.; Parkin, A.; Davies, G. J.; Walton, P. H. The Copper Active Site of CBM33 Polysaccharide Oxygenases. J. Am. Chem. Soc. 2013, 135 (16), 6069-6077. https://doi.org/10.1021/ja402106e.

(4) Aachmann, F. L.; Sørlie, M.; Skjåk-Bræk, G.; Eijsink, V. G. H.; Vaaje-Kolstad, G. NMR Structure of a Lytic Polysaccharide Monooxygenase Provides Insight into Copper Binding, Protein Dynamics, and Substrate Interactions. PNAS 2012, 109 (46), 18779-18784. https://doi.org/10.1073/pnas.1208822109.

(5) Cao, L.; Caldararu, O.; Rosenzweig, A. C.; Ryde, U. Quantum Refinement Does Not Support Dinuclear Copper Sites in Crystal Structures of Particulate Methane Monooxygenase. Angew. Chem. Int. Ed. 2018, 57 (1), 162-166. https://doi.org/10.1002/anie.201708977.

(6) Ross, M. O.; MacMillan, F.; Wang, J.; Nisthal, A.; Lawton, T. J.; Olafson, B. D.; Mayo, S. L.; Rosenzweig, A. C.; Hoffman, B. M. Particulate Methane Monooxygenase Contains Only Mononuclear Copper Centers. Science 2019, 364 (6440), 566-570. https://doi.org/10.1126/science.aav2572.

(7) Garcia-Bosch, I. Copper-Catalyzed Oxidation of Alkanes under Mild Conditions. Synlett 2017, 28 (11), 1237-1243. https://doi.org/10.1055/s-0036-1590202.

(8) Concia, A. L.; Beccia, M. R.; Orio, M.; Ferre, F. T.; Scarpellini, M.; Biaso, F.; Guigliarelli, B.; Réglier, M.; Simaan, A. J. Copper Complexes as Bioinspired Models for Lytic Polysaccharide Monooxygenases. Inorg. Chem. 2017, 56 (3), 1023-1026. https://doi.org/10.1021/acs.inorgchem.6b02165.

(9) Garcia-Bosch, I.; Siegler, M. A. Copper-Catalyzed Oxidation of Alkanes with H2O2 under a Fenton-like Regime. Angew. Chem. Int. Ed. 2016, 55 (41), 12873-12876. https://doi.org/10.1002/anie.201607216.

(10) Elwell, C. E.; Gagnon, N. L.; Neisen, B. D.; Dhar, D.; Spaeth, A. D.; Yee, G. M.; Tolman, W. B. Copper-Oxygen Complexes Revisited: Structures, Spectroscopy, and Reactivity. Chem. Rev. 2017, 117 (3), 2059-2107. https://doi.org/10.1021/acs.chemrev.6b00636.

(11) Schnödt, J.; Manzur, J.; García, A.-M.; Hartenbach, I.; Su, C.-Y.; Fiedler, J.; Kaim, W. Coordination of a Hemilabile N,N,S Donor Ligand in the Redox System [CuL2]+/2+, L = 2Pyridyl-N-(2'-Alkylthio-phenyl)Methyleneimine. Eur. J. Inorg. Chem. 2011, No. 9, 14361441. https://doi.org/10.1002/ejic.201001183.

(12) Seth, D. K.; Bhattacharya, S. Copper(I) Complexes of N-(Aryl)Pyridine-2-Aldimines: Spectral, Electrochemical and Catalytic Properties. Polyhedron 2011, 30 (15), 2438-2443. https://doi.org/10.1016/j.poly.2011.05.037. 
(13) Lagerspets, E.; Lagerblom, K.; Heliövaara, E.; Hiltunen, O.-M.; Moslova, K.; Nieger, M.; Repo, T. Schiff Base Cu(I) Catalyst for Aerobic Oxidation of Primary Alcohols. Mol. Catal. 2019, 468, 75-79. https://doi.org/10.1016/j.mcat.2019.02.003.

(14) Hamblin, J.; Childs, L. J.; Alcock, N. W.; Hannon, M. J. Directed One-Pot Syntheses of Enantiopure Dinuclear Silver(I) and Copper(I) Metallo-Supramolecular Double Helicates. J. Chem. Soc., Dalton Trans. 2002, No. 2, 164-169. https://doi.org/10.1039/B106987J.

(15) Tanaka, T.; Sunatsuki, Y.; Suzuki, T. Synthesis and Magnetic Properties of Tetrahedral Tetranuclear Iron(II) Complexes with Bis(Bidentate)-Type Schiff Bases Containing Imidazole Groups. Inorg. Chim. Acta 2020, 502, 119373. https://doi.org/10.1016/j.ica.2019.119373.

(16) Mimura, M.; Matsuo, T.; Nakashima, T.; Matsumoto, N. Zigzag-Chain and CyclicTetrameric Compounds Derived by Deprotonation of Mononuclear Copper(II) Complexes with N,N`-Bis(2-Substituted-Imidazol-4-Ylmethyl- Idene)-1,4-Diaminobutane (2Substituent $=\mathrm{H}, \mathrm{Me})$ : Synthesis, Characterization, Structure, Substituent Effect, and Interconvertibility. Inorg. Chem. 1998, $37 \quad$ (14), 3553-3560. https://doi.org/10.1021/ic971609c.

(17) Gudmundsson, M.; Kim, S.; Wu, M.; Ishida, T.; Momeni, M. H.; Vaaje-Kolstad, G.; Lundberg, D.; Royant, A.; Ståhlberg, J.; Eijsink, V. G. H.; Beckham, G. T.; Sandgren, M. Structural and Electronic Snapshots during the Transition from a $\mathrm{Cu}(\mathrm{II})$ to $\mathrm{Cu}(\mathrm{I}) \mathrm{Metal} \mathrm{Center}$ of a Lytic Polysaccharide Monooxygenase by X-Ray Photoreduction. J. Biol. Chem. 2014, 289 (27), 18782-18792. https://doi.org/10.1074/jbc.M114.563494.

(18) Donoghue, P. J.; Gupta, A. K.; Boyce, D. W.; Cramer, C. J.; Tolman, W. B. An Anionic, Tetragonal Copper(II) Superoxide Complex. J. Am. Chem. Soc. 2010, 132 (45), 1586915871. https://doi.org/10.1021/ja106244k.

(19) Fukatsu, A.; Morimoto, Y.; Sugimoto, H.; Itoh, S. Modelling a 'Histidine Brace' Motif in Mononuclear Copper Monooxygenases. Chem. Commun. 2020, 56 (38), 5123-5126. https://doi.org/10.1039/D0CC01392G.

(20) Vaaje-Kolstad, G.; Westereng, B.; Horn, S. J.; Liu, Z.; Zhai, H.; Sørlie, M.; Eijsink, V. G. H. An Oxidative Enzyme Boosting the Enzymatic Conversion of Recalcitrant Polysaccharides. Science 2010, 330 (6001), 219-222. https://doi.org/10.1126/science.1192231.

(21) Becker, J.; Gupta, P.; Angersbach, F.; Tuczek, F.; Näther, C.; Holthausen, M. C.; Schindler, S. Selective Aromatic Hydroxylation with Dioxygen and Simple Copper Imine Complexes. Chem. Eur. J. 2015, 21 (33), 11735-11744. https://doi.org/10.1002/chem.201501003.

(22) Lerch, M.; Weitzer, M.; Stumpf, T.-D. J.; Laurini, L.; Hoffmann, A.; Becker, J.; Miska, A.; Göttlich, R.; Herres-Pawlis, S.; Schindler, S. Kinetic Investigation of the Reaction of Dioxygen with the Copper(I) Complex $[\mathrm{Cu}(\mathrm{PimiPr} 2)(\mathrm{CH} 3 \mathrm{CN})] \mathrm{CF} 3 \mathrm{SO} 3$ PimiPr2 = Tris[2(1,4-Diisopropylimidazolyl)]Phosphine. Eur. J. Inorg. Chem. 2020, 2020 (33), 3143-3150. https://doi.org/10.1002/ejic.202000462.

(23) Lennox, A. J. J.; Fischer, S.; Jurrat, M.; Luo, S.-P.; Rockstroh, N.; Junge, H.; Ludwig, R.; Beller, M. Copper-Based Photosensitisers in Water Reduction: A More Efficient In Situ Formed System and Improved Mechanistic Understanding. Chemistry - A European Journal 2016, 22 (4), 1233-1238. https://doi.org/10.1002/chem.201503812.

(24) Zhang, Y.; Heberle, M.; Wächtler, M.; Karnahl, M.; Dietzek, B. Determination of Side Products in the Photocatalytic Generation of Hydrogen with Copper Photosensitizers by 
Resonance Raman Spectroelectrochemistry. RSC Adv. 2016, 6 (107), 105801-105805. https://doi.org/10.1039/C6RA21469J.

(25) Rosiak, D.; Okuniewski, A.; Chojnacki, J. Novel Complexes Possessing Hg-(Cl, Br, I) ․OC Halogen Bonding and Unusual Hg2S2(Br/I)4 Kernel. The Usefulness of T4' Structural Parameter. Polyhedron 2018, 146, 35-41. https://doi.org/10.1016/j.poly.2018.02.016.

(26) Okuniewski, A.; Rosiak, D.; Chojnacki, J.; Becker, B. Coordination Polymers and Molecular Structures among Complexes of Mercury(II) Halides with Selected 1-Benzoylthioureas. Polyhedron 2015, 90, 47-57. https://doi.org/10.1016/j.poly.2015.01.035.

(27) Hylland, K. T.; Øien-Ødegaard, S.; Tilset, M. The Suzuki-Miyaura Cross-Coupling as the Key Step in the Synthesis of 2-Aminobiphenyls and 2,2'-Diaminobiphenyls: Application in the Synthesis of Schiff Base Complexes of Zn. Eur. J. Org. Chem. 2020, No. 27, 4208-4226. https://doi.org/10.1002/ejoc.202000599.

(28) Hylland, K. T.; Øien-Ødegaard, S.; Heyn, R. H.; Tilset, M. Zinc Schiff Base Complexes Derived from 2,2'-Diaminobiphenyls: Solution Behavior and Reactivity towards Nitrogen Bases. Eur. J. Inorg. Chem. 2020, No. 38, 3627-3643. https://doi.org/10.1002/ejic.202000589.

(29) Sheldrick, G. M. Crystal Structure Refinement with SHELXL. Acta Cryst C 2015, 71 (1), 38. https://doi.org/10.1107/S2053229614024218.

(30) Sheldrick, G. M. SHELXT - Integrated Space-Group and Crystal-Structure Determination. Acta Cryst A 2015, 71 (1), 3-8. https://doi.org/10.1107/S2053273314026370.

(31) Dolomanov, O. V.; Bourhis, L. J.; Gildea, R. J.; Howard, J. a. K.; Puschmann, H. OLEX2: A Complete Structure Solution, Refinement and Analysis Program. J Appl Cryst 2009, 42 (2), 339-341. https://doi.org/10.1107/S0021889808042726.

(32) Allen, F. H.; Johnson, O.; Shields, G. P.; Smith, B. R.; Towler, M. CIF Applications. XV. EnCIFer: A Program for Viewing, Editing and Visualizing CIFs. J Appl Cryst 2004, 37 (2), 335-338. https://doi.org/10.1107/S0021889804003528. 